\title{
AUMENTO DE 5\% EN LA COTIZACIÓN PREVISIONAL Y SU IMPACTO EN LAS PENSIONES
}

\author{
Fernando López \\ Ph.D. en Finanzas, John M. Olin Business School, Washington University in St. Louis \\ Académico FEN
}

\begin{abstract}
Una de las principales medidas propuestas por el Gobierno para mejorar las pensiones es aumentar gradualmente la tasa de cotización previsional en cinco puntos porcentuales, con cargo exclusivo al empleador. Esta iniciativa ha sido objeto de un amplio debate en relación al destino de los fondos, la gradualidad de implementación, su impacto en el mercado laboral y, recientemente, respecto a su posible impacto en las pensiones. En este artículo se analizará este último punto, enfatizando los principales factores que determinarán el impacto de la mayor tasa de cotización previsional en trabajadores y pensionados.

El efecto de un aumento de la tasa de cotización en la pensión de una persona depende de múltiples variables, como por ejemplo, el destino de las cotizaciones (cuentas individuales o fondo de reparto), número de años en que los trabajadores contribuyan una mayor tasa y la rentabilidad de los fondos en que se invierta esta mayor cotización. Para ilustrar la heterogeneidad del impacto en la pensión de personas con distintas caracteristicas, es conveniente recordar que el monto de las pensiones depende de cuatro factores: del ahorro que esta logre acumular durante su vida laboral; la rentabilidad de sus ahorros previsionales; el aporte estatal a su pensión y la expectativa de vida, que corresponde a una estimación del número de años que las personas vivirán luego de jubilarse (Figura 1).
\end{abstract}

Figura 1

Determinantes de las pensiones

Pensión $=\frac{(\text { Ahorro Previsional } \times \text { Rentabilidad }+ \text { Aporte Estatal })}{(\text { Expectativa de vida })}$

Conceptualmente, el mayor ahorro previsional impactará más a los trabajadores jóvenes, quienes tendrán una mayor tasa de ahorro por un periodo más extenso. Para cuantificar los efectos posibles, se presentan estimaciones del impacto que tendrá la mayor cotización en las pensiones de trabajadores pertenecientes a distintos tramos de edad. Por simplicidad, el análisis se divide en dos partes. Primero, se supone que el aumento en la cotización será destinado por completo a las cuentas individuales de los trabajadores y que estos no la percibirán como un impuesto a sus ingresos laborales'. Segundo, se analiza el caso en que una parte de la cotización adicional se destine a un fondo de reparto (o solidario).

ANÁLISIS DE IMPACTO SI EL AUMENTO DE COTIZACIÓN SE DESTINA POR COMPLETO A LAS CUENTAS INDIVIDUALES

A continuación se analiza un aumento en la tasa de cotización en hombres de 20,30,40, 50 y 60 años bajo los supuestos de que todos comenzaron a cotizar a los 20 años, no tienen lagunas laborales, su ingreso se mantiene constante a lo largo de su vida y la rentabilidad de sus fondos de pensiones será igual a 5\% (similar a la obtenida por el Fondo $\mathrm{C}$ desde que se iniciaron los multifondos). A modo de ejemplo, se considera un trabajador de 40 años que ha trabajado ininterrumpidamente desde los 20 años con un nivel de ingreso constante. A partir de los 40 años, su tasa de cotización aumenta progresivamente de acuerdo a distintos escenarios posibles de implementación de la política. El análisis de impacto para el caso de las mujeres entrega resultados similares cuantitativa y cualitativamente. Por esta razón, solo se presentan resultados para los hombres.

Los resultados de la primera columna de la Tabla 1 asumen que la tasa de cotización aumenta a un ritmo de 0,5\% anual. En este caso, se aprecia que la pensión aumentará en un 39,2\% para quienes experimenten el aumento de cotización a los 20 años. La magnitud de este impacto se reduce a $21,5 \%$ para quienes tengan 30 años y a 10,7\% para quienes tengan 40 años al momento en que aumente la tasa de cotización. Para personas de más de 50 años, el aumento en la pensión es inferior a 5\%. La segunda columna considera que la tasa de cotización aumenta en un 1\% anual. En este caso, la pensión aumentaria en un $44,8 \%$ para los trabajadores que experimentan el alza en sus cotizaciones a los 20 años y en $24,9 \%$ para quienes tengan 30 años al momento en que aumenten sus cotizaciones. A partir de los 40 años, el 
impacto caería a $12,8 \%$ y a menos de $6 \%$ para trabajadores que sean mayores a 50 años al momento en que aumente la tasa de cotización. Por último, también se observa un mayor impacto en las pensiones cuando la medida se implementa de manera inmediata. El impacto sobre trabajadores de 20 años es igual a $50 \%$ y de $28 \%$ para trabajadores de 30 años. Sin embargo, al igual que en los casos anteriores, el impacto se reduce a 15\% para trabajadores de 40 años y a menos de 7\% para trabajadores mayores a 50 años.

TABLA 1.

AUMENTO PORCENTUAL ESPERADO EN EL NIVEL DE PENSIÓN ASOCIADO A UN AUMENTO DE 5\% EN LA TASA DE COTIZACIÓN PREVISIONAL PARA DISTINTOS TRAMOS DE EDAD Y GRADUALIDAD DE IMPLEMENTACIÓN

\begin{tabular}{cccc}
\hline $\begin{array}{c}\text { Edad a la que } \\
\text { aumenta cotización }\end{array}$ & $\begin{array}{c}(1) \\
\text { 0,5 por año }\end{array}$ & $\begin{array}{c}(2) \\
\text { 1\% por año }\end{array}$ & $\begin{array}{c}(3) \\
5 \% \text { inmediato }\end{array}$ \\
\hline 20 años & 39.2 & 44.8 & 50.0 \\
30 años & 21.5 & 24.9 & 28.0 \\
40 años & 10.7 & 12.8 & 14.7 \\
50 años & 4.2 & 5.4 & 6.6 \\
60 años & 0.5 & 1.0 & 1.7 \\
\hline
\end{tabular}

IMPACTO EN PENSIONES FINANCIADAS CON CAPITALIZACIÓN INDIVIDUAL CUANDO UNA FRACCIÓN DE LA COTIZACIÓN SE DESTINA A UN FONDO DE REPARTO

A continuación se cuantifica el aumento porcentual esperado en el nivel de pensión cuando una parte del 5\% adicional se distribuye a un fondo de reparto. La Tabla 2 presenta los resultados bajo el escenario descrito anteriormente, asumiendo aumentos de 0,5\% anual en la tasa de cotización y que los primeros incrementos serán destinados a un fondo de reparto. La primera columna presenta el caso base, en que un 100\% de la mayor cotización se destina a la cuenta de capitalización individual. Los resultados muestran que al destinar un 0,5\% del aumento en la cotización a un pilar de reparto, el impacto en las pensiones se reduce en menos de 3 puntos porcentuales para trabajadores mayores a 30 años y en 5 puntos porcentuales para trabajadores de 20 años. Por su parte, la tercera columna muestra que cuando un $1 \%$ de la mayor cotización se destina a un pilar solidario, el aumento esperado en el nivel de pensión de los trabajadores de 20 años cae en un 25\%, de $39 \%$ a $29 \%$. El impacto se reduce en 5,5 puntos porcentuales para trabajadores de 30 años y en menos de 3 puntos porcentuales para mayores a 40 años. En el caso que un $2 \%$ de la cotización adicional se destine al fondo solidario, los más afectados serian los trabajadores que tengan más de 50 años, quienes perderian más de $50 \%$ del aumento que obtendrian en su pensión si sus ahorros no fueran a un fondo de reparto, y los trabajadores de 60 años, que perderian un 94\% de dicho aumento.
TABLA 2.

AUMENTO PORCENTUAL ESPERADO EN EL NIVEL DE PENSIÓN CUANDO PARTE DEL 5\% ADICIONAL SE DISTRIBUYE A UN PILAR SOLIDARIO (O DE REPARTO)

\begin{tabular}{cccccc}
\hline $\begin{array}{c}\text { Edad a la que } \\
\text { aumenta cotización }\end{array}$ & $(1)$ & $(2)$ & $(3)$ & $(4)$ & $(5)$ \\
\hline 20 años & $0.0 \%$ & $0.5 \%$ & $1.0 \%$ & $1.5 \%$ & $2.0 \%$ \\
30 años & 39.2 & 34.2 & 29.5 & 25.0 & 23.6 \\
40 años & 21.5 & 18.6 & 16.0 & 13.6 & 12.7 \\
50 años & 10.7 & 9.2 & 7.8 & 6.6 & 6.1 \\
60 años & 4.2 & 3.5 & 2.9 & 2.4 & 2.1 \\
& 0.5 & 0.3 & 0.2 & 0.1 & 0.0 \\
\hline
\end{tabular}

IMPACTO DEL FONDO DE REPARTO EN LAS PENSIONES ACTUALES

Con la reforma, los recursos que no son destinados a las cuentas de capitalización individual serán destinados a un fondo solidario que tiene por objeto complementar las "bajas" pensiones de los jubilados actuales. El impacto de esta medida en las pensiones de los jubilados depende del porcentaje de aumento en la cotización destinado al pilar de reparto y las distorsiones que esta produzca en el mercado laboral. Para determinar una cota superior del impacto que tendría esta medida se asume que los trabajadores no perciben la mayor cotización ni la parte destinada a reparto como un impuesto al trabajo. Específicamente, la Tabla 3 muestra el impacto asociado a destinar entre un $0,5 \%$ y un $2 \%$ de la cotización adicional al pago de pensiones de los 1,2 millones de pensionados actuales. El ejercicio considera como fuente de financiamiento las cotizaciones adicionales de los 5,1 millones de cotizantes que tenían un ingreso imponible promedio de $\$ 707$ mil a septiembre de 2016. Se aprecia que por cada 0,5\% de aumento en la cotización adicional destinado a un esquema de reparto, las pensiones de los jubilados actuales aumentaría en 7,3\%. De esta manera, destinando un $1 \%$ al fondo de reparto las pensiones actuales aumentarian un $14,7 \%$ y destinando un 1,5\% a este fondo las pensiones actuales aumentarian en un 22\%. Cabe señalar que estos resultados se sostienen en que a septiembre de 2016 teníamos 4,3 cotizantes por cada pensionado. A 2050 se estima que habrá 1,8 cotizantes por cada pensionado, una cifra que ilustra que el efecto de esta iniciativa se irá reduciendo en el tiempo. A modo de ejemplo, si hoy tuviéramos 1,8 cotizantes por cada pensionado, el impacto del fondo de reparto en las pensiones de los jubilados actuales se reduciría en más de $50 \%$.

TABLA 3.

IMPACTO DE UNA MAYOR COTIZACIÓN EN LAS PENSIONES DE LOS JUBILADOS ACTUALES

\begin{tabular}{ccc}
\hline $\begin{array}{c}\text { \% de aumento de } \\
\text { cotizacion destinado } \\
\text { a reparto }\end{array}$ & $\begin{array}{c}\text { Recursos destinados } \\
\text { a pensiones } \\
\text { (MM\$) }\end{array}$ & $\begin{array}{c}\text { \% Aumento en } \\
\text { pensiones }\end{array}$ \\
\hline $0.5 \%$ & 18,262 & 7.3 \\
$1.0 \%$ & 36,524 & 14.7 \\
$1.5 \%$ & 54,786 & 22.0 \\
$2.0 \%$ & 73,048 & 29.4 \\
\hline
\end{tabular}

Fuente: Elaboración propia con datos de la Superintendencia de Pensiones.. 